Chinese Journal of Organic Chemistry

\title{
贝克曼重排的研究新进展
}

\author{
张健*,a,c 刘园园 ${ }^{b}$ 冯维春 ${ }^{c}$ 武玉民 ${ }^{a}$ \\ $\left({ }^{a}\right.$ 青岛科技大学化工学院 青岛 266042) \\ ( ${ }^{b}$ 青岛科技大学化学与分子工程学院 青岛 266042)

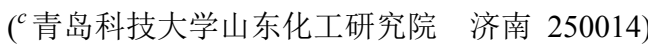

\begin{abstract}
摘要 酰胺类化合物广泛存在于天然产物、药物和生物活性分子中, 酰胺键的形成反应是有机化学最重要的反应之一, 贝克曼重排反应是合成伯酰胺和仲酰胺的重要方法. 近年来, 许多新的催化体系被建立, 包括从醠、酮甚至是醇出发的 一锅法反应. 主要综述了近五年来贝克曼重排的研究进展及其应用. 关键词 贝克曼重排; 酰胺; 合成; 肜
\end{abstract}

\section{Recent Progress in Beckmann Rearrangement}

\author{
Zhang, Jian ${ }^{*, a, c} \quad$ Liu, Yuanyuan $^{b} \quad$ Feng, Weichun $^{c} \quad$ Wu, Yumin ${ }^{a}$
}

$\left({ }^{a}\right.$ College of Chemical Engineering, Qingdao University of Science \& Technology, Qingdao 266042)

$\left({ }^{b}\right.$ College of Chemistry \& Molecular Engineering, Qingdao University of Science \& Technology, Qingdao 266042)

$\left({ }^{c}\right.$ Chemical Technology Academy of Shandong Province, Qingdao University of Science \& Technology, Qingdao 250014)

\begin{abstract}
Amide motifs are prevalent structures found in natural products, pharmaceuticals, and bioactive compounds. Amide bond formations are the most important transformations in organic chemistry. Beckmann rearrangement is a significant method for the synthesis of primary and secondary amides. Recently, diversified new catalytic protocols were developed, including one-pot reaction from the corresponding aldehydes and ketones, or even from alcohols. The progress and applications of Beckmann rearrangement in recent five years are reviewed.
\end{abstract}

Keywords Beckmann rearrangement; amides; synthesis; oximes

酰胺是有机化学和生物化学最重要的一类结构单 元, 在医药、农药及材料领域具有广泛的用途 ${ }^{[1]}$. 合成酰 胺最常用的方法是用羧酸衍生物, 如酰氯、酸酐或酯与 胺反应, 或者是用羧酸与胺在当量缩合剂(如羰基咪唑) 的存在下直接反应 ${ }^{[2]}$. 尽管这些合成方法非常高效, 但 是需要使用价格高、毒性大的原料, 或者产生大量副产 物, 原子经济性差, 对环境不友好, 不符合绿色化学的 要求. 因此, 建立绿色、可持续性的合成新策略构筑酰 胺键是当今有机合成的迫切需求.

贝克曼重排反应 ${ }^{[3]}$, 由于其操作简单、高选择性及 原子经济性, 一直是合成酰胺的首选方法, 可以非常方 便地制备各种结构的伯酰胺和仲酰胺(Eq. 1). 贝克曼重 排也已应用于大规模的工业化生产, 如利用环已酮肜合
成尼龙 6 的前体 $\varepsilon$-己内酰胺(Eq. 2).
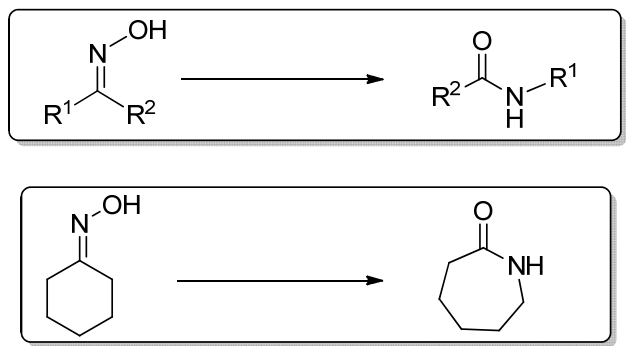

贝克曼重排的反应机理是与肜羟基处于反式的基 团从碳原子迁移到氮原子上，反应一般是在布朗斯台德 酸的存在下进行 ${ }^{[4]}$. 芳基和烷基都可以顺利地发生迁移, 将酮肜转化为 $N$-取代的酰胺, 而醛肜的重排反应得到 的产物一般是腈不是酰胺 ${ }^{[5]}$. 近年来, 金属催化的贝克

\footnotetext{
* Corresponding author. E-mail: jian8552@163.com

Received September 26, 2018; revised November 6, 2018; published online December 17, 2018.

Project supported by the Science and Technology Development Project in University of Shandong Province (No. J18KA102), the Natural Science Foundation of Shandong Province of China (No. ZR201807060818).

山东省高校科研计划(No. J18KA102)和山东省自然科学基金(No. ZR201807060818)资助项目.
} 
曼重排反应突破了这一局限性 ${ }^{[6]}$. 另外，从酮或醛甚至 是醇出发的一锅法反应也被不断报道, 使反应变得更加 高效. 从金属催化、无金属催化、重排的应用三个方面 全面综述了近 5 年贝克曼重排的新进展.

\section{1 金属催化的贝克曼重排}

醛肜可以在许多过渡金属(如 $\mathrm{Ru}^{[7]} 、 \mathrm{Rh}^{[8]} 、 \mathrm{Pd}^{[9]}$ 、 $\mathrm{Cu}^{[10]} 、 \mathrm{Co}^{[11]} 、 \mathrm{Fe}^{[12]}$ 等)的催化下转化为伯酰胺, 反应的 机理与传统酸催化机理明显不同 ${ }^{[13]}$, 金属参与了失水 得到中间体腈, 以及水重新加成生成产物的全过程.

\section{1 钉催化的贝克曼重排}

近年来, 钉催化下醛肜的贝克曼重排成为研究的热 点 ${ }^{[14]}$. Cadierno 课题组 ${ }^{[15]}$ 做了系统的研究工作. 2013 年 该小组用易得的二价钓催化剂实现醛肜一锅法合成伯 酰胺的贝克曼重排, 该反应官能团耐受性好, 以水作溶 剂, 具有绿色环保的优点 ${ }^{[16]}$. 同年, 该课题组 ${ }^{[17]}$ 还报道 了另外一例二价钉催化的重排反应, 用醛与盐酸差弪胺在 水中直接反应形成酰胺. 最近, Cadierno 课题组 ${ }^{[18]}$ 又报 道了一种新型含磷肜配体的 $\mathrm{Ru}$ 配合物, 结构通过 $\mathrm{X}$ 单 晶衍射确定. 该化合物能够高效地催化醛肜的贝克曼重 排反应, 反应在水相中进行, 具有较高的收率和较为广 泛的底物范围(Eq. 3).
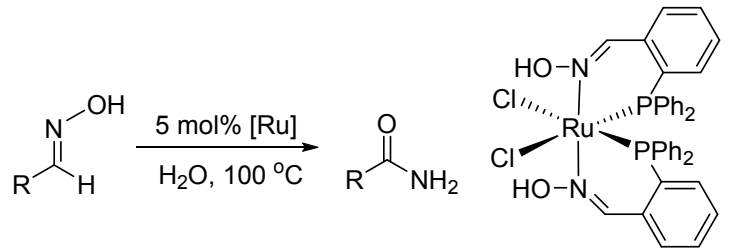<smiles>NC(=O)c1ccccc1</smiles><smiles>NC(=O)c1ccc([N+](=O)[O-])cc1</smiles><smiles>NC(=O)c1cccnc1</smiles><smiles>NC(=O)C1CCCCC1</smiles><smiles>NC(=O)/C=C/c1ccccc1</smiles>

2015 年, García-Garrido 课题组 ${ }^{[19]}$ 使用商品化的四 价钓作催化剂实现了醛肟的贝克曼重排反应. 该方法使 用水/甘油作溶剂, 无需添加催化剂, 底物范围广, 各种 芳香、脂肪以及 $\alpha, \beta$-不饱和醛肜都可以反应得到伯酰胺. 另外，钓催化剂可以回收再利用(Scheme 1).

2016 年, 宋毛平课题组 ${ }^{[20]}$ 使用钓螯合物同样实现 了醛肟向伯酰胺的转化, 催化剂的用量只需 $0.5 \mathrm{~mol} \%$, 该反应用水作溶剂且不需要惰性气体保护, 操作简单. 还可以用醛和盐酸羟胺在钓催化下一步直接合成伯酰 胺, 除了芳醛外, 共轭醛和脂肪醛也可以发生反应

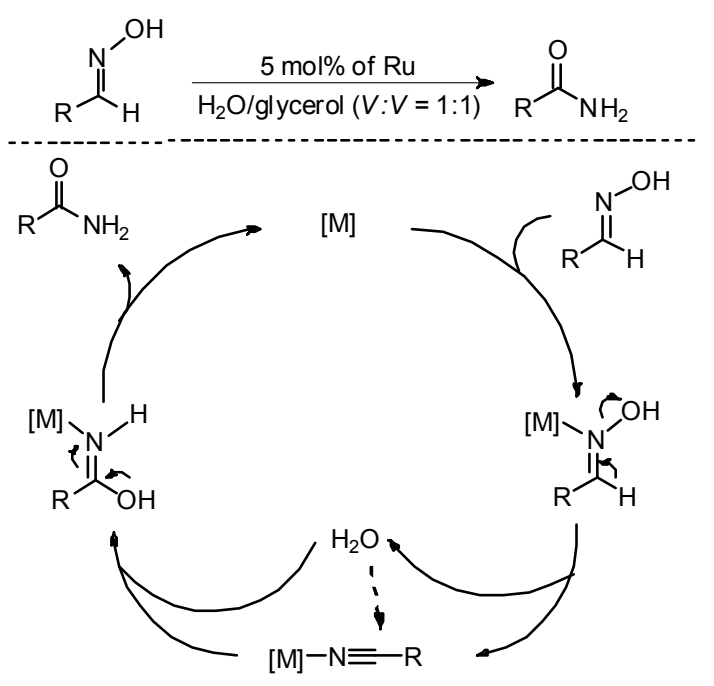

图式 1 钉催化的贝克曼重排

Scheme 1 Ru-catalyzed Beckmann rearrangement

(Scheme 2).

\section{2 铁催化的贝克曼重排}

相比于其他金属，铁催化剂具有便宜易得的优点. 2011 年, Chakraborty 课题组 ${ }^{[21]}$ 在水相中实现了首例铁催 化的醛肟向伯酰胺的转化. 在此基础上，该课题组 ${ }^{[22]}$ 发 展了由醇一锅法直接合成伯酰胺的新方法(Eq. 4). 首先 醇在碘-TEMPO 的作用下原位氧化为酫，然后与盐酸着 胺反应得到肜, 最后在三氯化铁催化下重排得到伯酰 胺. 该方法简单高效，芳香醇、杂芳香醇、脂肪醇以及 烯丙基醇都可以发生反应，以较高的收率得到产物酰 胺, 未观察到副产物的生成.
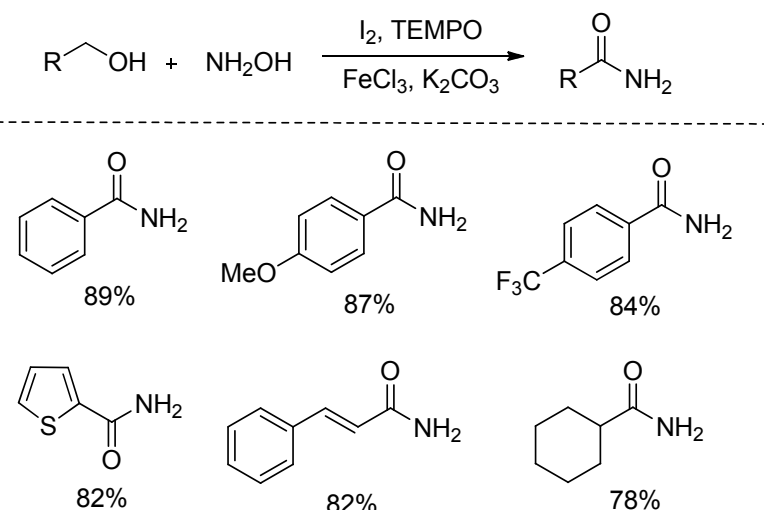<smiles>NC(=O)/C=C/c1ccccc1</smiles><smiles>NC(=O)C1CCCCC1</smiles>

2015 年, Kapoor 课题组 ${ }^{[23]}$ 使用 $\mathrm{FeCl}_{3} \cdot 6 \mathrm{H}_{2} \mathrm{O}$ 为催化 剂, 将酮在盐酸着弪胺存在下直接经贝克曼重排转化为酰 胺. 该方法最大亮点是无溶剂固相反应(Eq. 5). 同年, Cook 课题组 ${ }^{[24]}$ 发展了一种铁催化贝克曼重排合成酰胺 的新方法(Eq. 6). 该方法条件温和，避免了使用强酸， 且不需要惰性气体保护, 不同结构的肜均可重排得到酰 胺. 

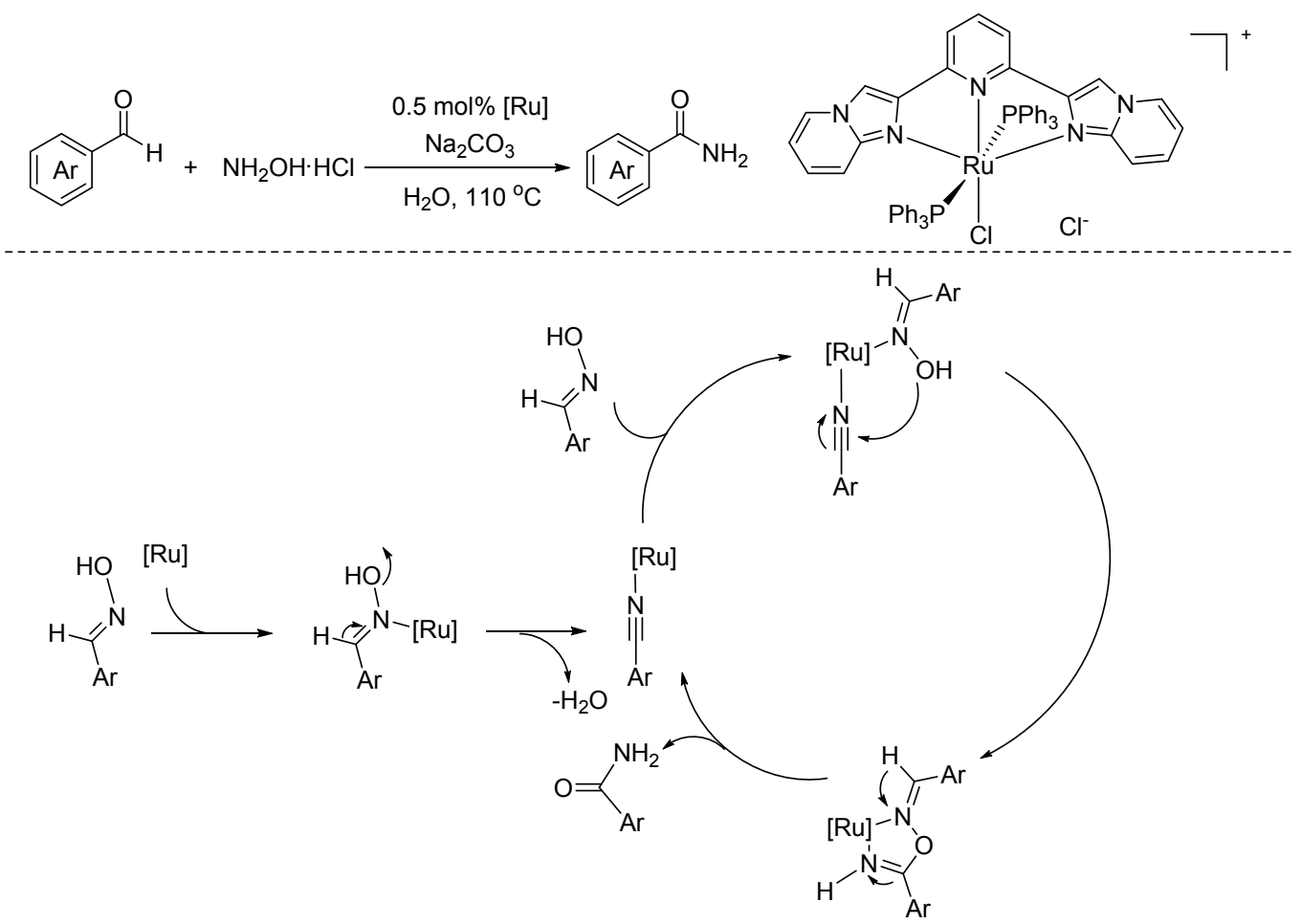

图式 2 钓催化的贝克曼重排

Scheme 2 Ru-catalyzed Beckmann rearrangemen

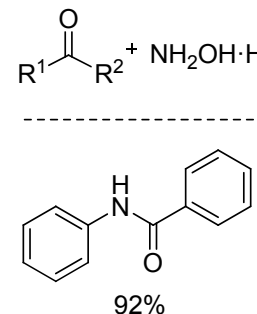

$92 \%$<smiles>O=C1CCCCCN1</smiles>

$72 \%$
$\underset{\mathrm{FeCl}_{3} \cdot 6 \mathrm{H}_{2} \mathrm{O}(10 \mathrm{~mol} \%)}{130{ }^{\circ} \mathrm{C}} \mathrm{R}^{2} \stackrel{\mathrm{I}}{\mathrm{N}}_{\mathrm{N}^{-}} \mathrm{R}^{1}$<smiles>CCC(=O)Nc1ccccc1</smiles>

$88 \%$<smiles>O=C1CCCc2ccccc2N1</smiles>

$70 \%$<smiles>CC(=O)Nc1cccc(O)c1</smiles><smiles>O=c1[nH]c2ccccc2c2nc3ccccc3nc12</smiles>

(6)<smiles>[Y20]c1ccc(NC(C)=O)cc1</smiles><smiles>CCC(=O)Nc1ccccc1</smiles>

$67 \%$<smiles>O=C1CCCCCC1</smiles>

$76 \%$<smiles>COc1cc(NC=O)cc(OC)c1OC</smiles><smiles>Cc1nc2ccccc2c2ccccc12</smiles>
$\mathrm{Pd}(\mathrm{OAc})_{2}$<smiles>CCCCOP(=O)(OCc1ccccc1)OC(C)C</smiles><smiles>CC(=O)Nc1ccccc1</smiles>
$\mathrm{ZnBr}_{2} / \mathrm{TFA}$ 体在醋酸钯催化下进行芳基化，接着在 Hendrickson 试 剂作用下，可以转化为菲啶衍生物.

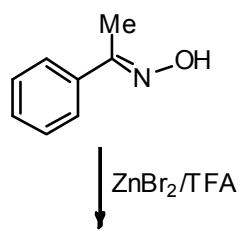<smiles></smiles><smiles>CC(=O)Nc1ccccc1P(C)(C)(OC(C)=O)c1ccccc1</smiles>

图式 3 锌催化的贝克曼重排

Scheme 3 Zn-catalyzed Beckmann rearrangement 
2016 年, Jacob 课题组 ${ }^{[26]}$ 报道了一种简单高效合成 新型有机硒取代酮肜的方法, 该类结构具有抗菌及抗氧 化活性, 在氯化锌的催化下酮肜进一步发生贝克曼重排 得到 $\alpha$ 硒代酰胺(Eq. 7).

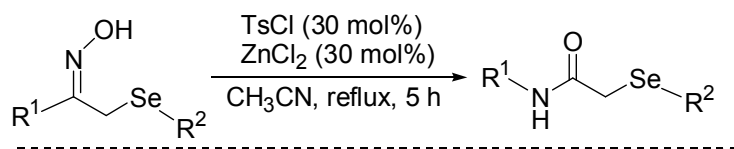<smiles>O=C(C[SeH]c1ccccc1)Nc1ccccc1</smiles><smiles>O=C(C[SeH]c1ccccc1)Nc1ccc([N+](=O)[O-])cc1</smiles>
$64 \%$<smiles>COc1ccc([Se][Se]C(=O)Nc2ccccc2)cc1</smiles><smiles>Cc1ccc(NC(=O)CSc2ccccc2)cc1</smiles>
$75 \%$<smiles>COc1ccccc1NC(=O)C[SeH]c1ccccc1</smiles>
$65 \%$<smiles>O=C(C[Se]c1ccc(F)cc1)Nc1ccccc1</smiles>

2017 年, Pathak 课题组 ${ }^{[27]}$ 利用壳聚糖负载的锌催化 剂成功地将酤一步转化为酰胺, 该多相催化剂通过过滤 即可回收, 且重复使用五次没有显着的催化活性损失 (Eq. 8).

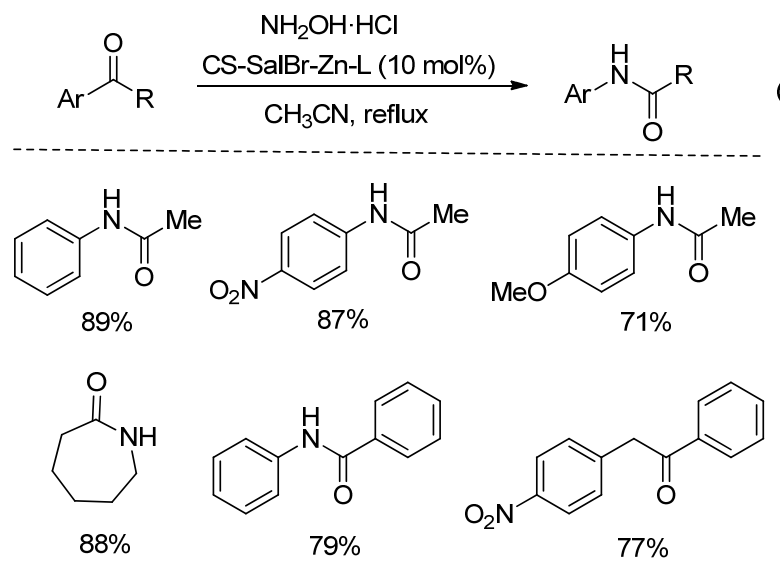

\section{4 铜催化的贝克曼重排}

铜催化的贝克曼重排已被系统的研究. Burri 课题 组 ${ }^{[28]}$ 通过制备 $\mathrm{Cu} / \mathrm{SBA}-15$ 催化剂, 在无溶剂中性条件下 实现了醛肜向酰胺的转变. Mondal 课题组 ${ }^{[29]}$ 发展了五 水硫酸铜催化的醛肜转化为伯酰胺的新方法. 2014 年, Jayaram 课题组 ${ }^{[30]}$ 发展了在水相中聚苯乙烯-Cu(II)络合 物催化醛肜转化为伯酰胺的方法.

Ramón 课题组 ${ }^{[31]}$ 发展了用醋酸铜作催化剂将醛直 接转化为伯酰胺的新方法, 该方法用水作溶剂、各种结 构的醛均可发生反应, 且催化剂只需经过简单萃取即可 回收利用(重复使用 10 次未见活性降低). 该反应条件温
和，收率高，无需使用配体和碱，粗产物只需重结晶即 可得到纯品(Eq. 9).

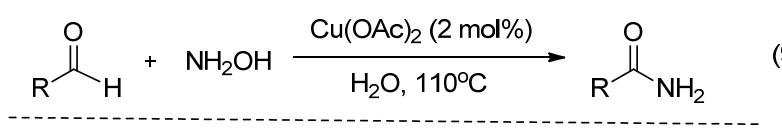<smiles>NC(=O)c1ccccc1</smiles>

$99 \%$<smiles>NC(=O)c1cccs1</smiles>

$99 \%$<smiles>NC(=O)c1ccc(Cl)cc1</smiles>

$98 \%$

$74 \%$<smiles>COc1ccc(C(N)=O)cc1</smiles>

$78 \%$<smiles>CC(C(N)=O)c1ccccc1</smiles>

$99 \%$<smiles>NC(=O)/C=C/c1ccccc1</smiles>

\section{5 钯催化的贝克曼重排}

2013 年, Pantos 课题组 ${ }^{[32]}$ 发展了一种二价钯作催化 剂将醛肜转化为酰胺的新方法, 该方法可在甲醇或水相 中反应. 最近, Cadierno 课题组 ${ }^{[33]}$ 用二苯基磷苯甲醛肜 与 $\mathrm{PdCl}_{2}(\mathrm{COD})$ 制备得到一种新型的二价钯配合物，结 构通过 $\mathrm{X}$ 单晶衍射确定. 该催化剂可以很好的催化醛肜 的贝克曼重排，以较好的收率得到伯酰胺，该方法底物 范围广, 用水作溶剂, 且不需要添加助催化剂(Scheme 4).

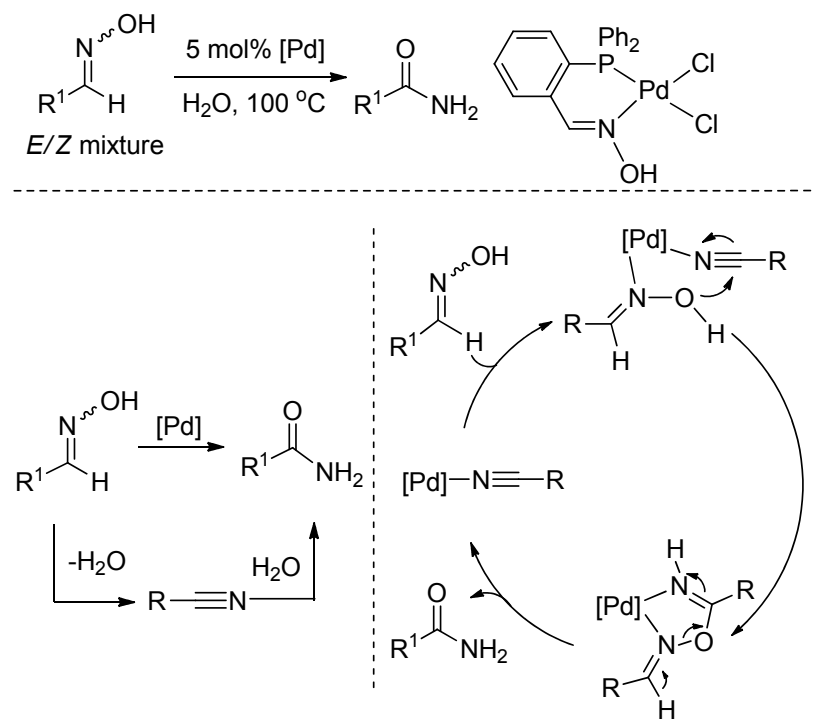

图式 4 钯催化的贝克曼重排

Scheme 4 Pd-catalyzed Beckmann rearrangement

\section{6 钙催化的贝克曼重排}

2018 年, McLaughlin 课题组 ${ }^{[34]}$ 报道了钙催化下酮肟 转化为酰胺的贝克曼重排, 该方法条件温和, 底物范围 广，并能够应用于雌酮类天然产物和阿奇霉素类药物的 合成. 另外，该方法可以从酮出发通过 “一锅法” 直接 得到酰胺. 反应可能的机理如 Scheme 5 所示. 


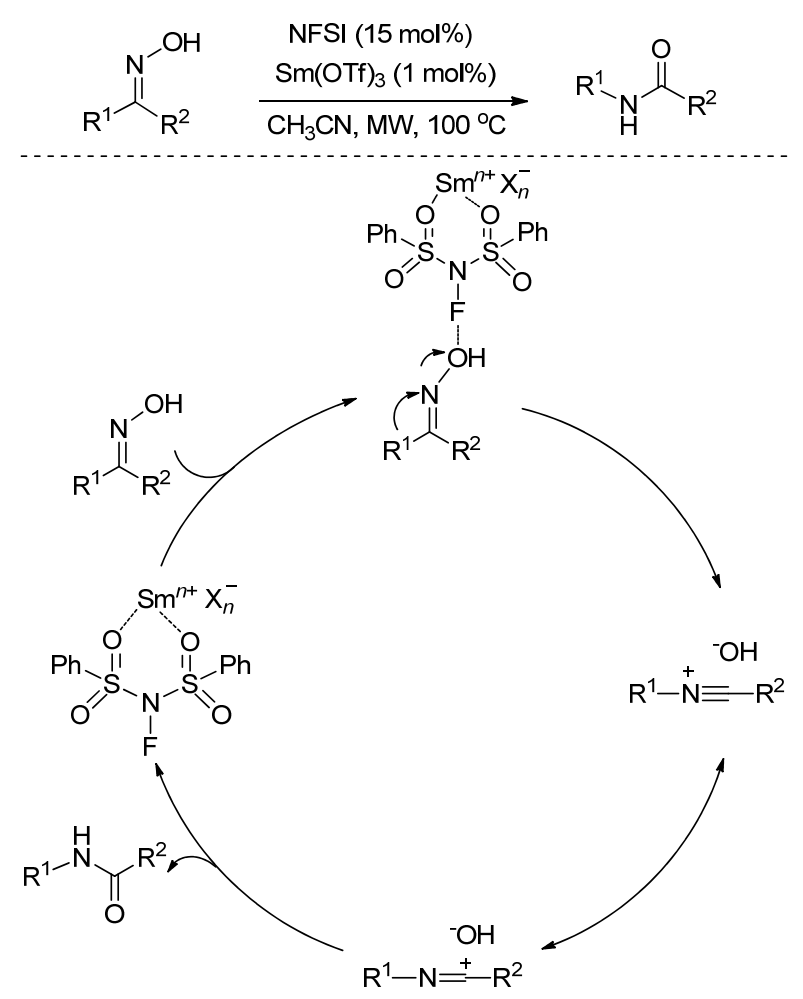

图式 5 钻催化肜贝克曼重排

Scheme 5 Ca-catalyzed Beckmann rearrangement

\section{7 钐催化的贝克曼重排}

刘永祥课题组 ${ }^{[35]}$ 发展了一种微波促进下 $N$-氟代双 苯磺酰胺(NFSI)/Sm 催化的贝克曼重排(Scheme 6). 作 者通过一系列控制实验和理论计算, 证实了钐和 NFSI 的作用模式. 路易斯酸与 NFSI 磺酰基的氧通过双齿配 位形成六元环过渡态，显著提高了氟原子的亲电活性， 使其更容易与肜羟基作用，协助重排发生.

\section{2 非金属催化的贝克曼重排}

\section{1 酸性条件下的贝克曼重排}

经典的贝克曼重排一般是用强的布朗斯台德酸催 化, 需要使用过量的强酸, 反应条件剧烈, 且产生大量 副产物腈. 近年来, 温和条件下酸催化的贝克曼重排被 大量报道 ${ }^{[36]}$. Kalkhambkar 课题组 ${ }^{[37]}$ 发展了用三氟甲磺 酸酐将酮肜高效转化为酰胺的新方法(Scheme 7). 该方 法无需使用添加剂或碱, 收率高, 底物范围广.

2015 年, Umanadh 课题组 ${ }^{[38]}$ 报道了一种掺杂硅胶的 高氯酸复合物, 能够高效地将酮肜转化为酰胺(Eq. 10). 该方法操作简单、条件温和且所用试剂便宜无毒.

2018 年, 张越涛课题组 ${ }^{[39]}$ 将木质素衍生物制备成 肜, 在二氯亚砜作用下, 乙腈作溶剂, 高效地发生贝克 曼重排得到酰胺(Scheme 8). 该方法操作简单, 反应速 度快, 收率高. 反应可能的机理是二氯亚砜与羟基作用

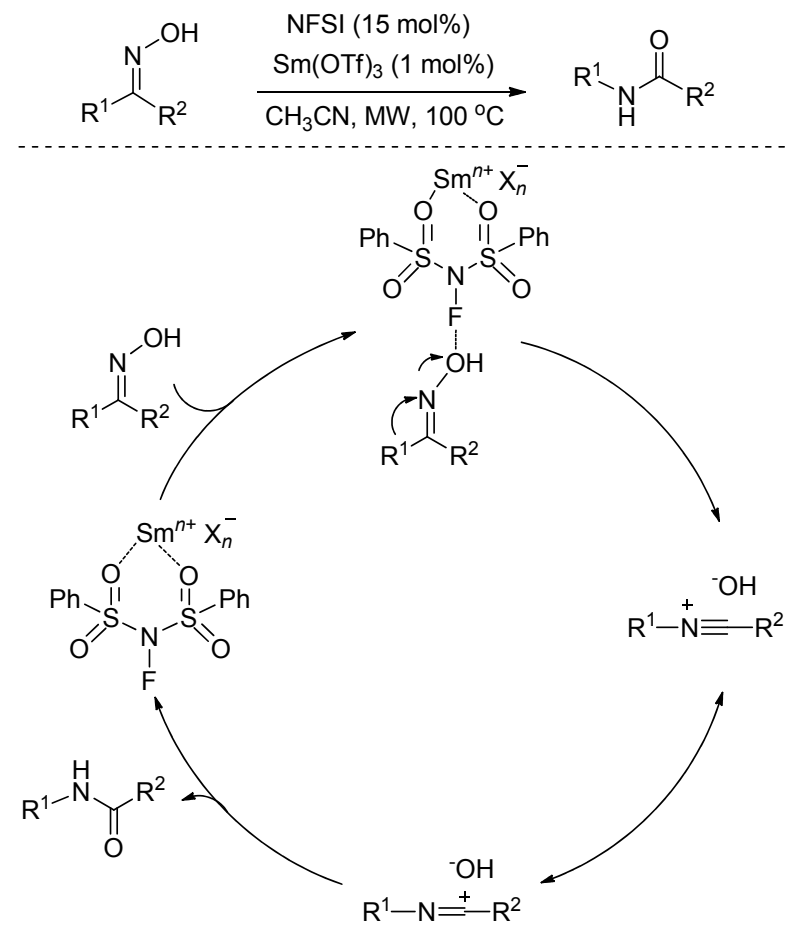

图式 6 钐催化的贝克曼重排

Scheme 6 Sm-catalyzed Beckmann rearrangement

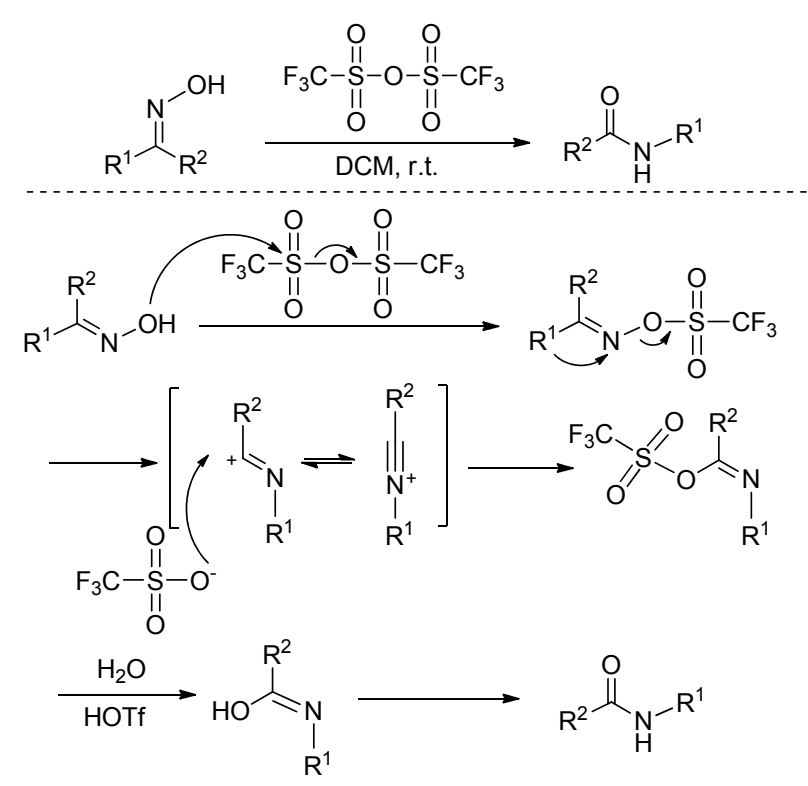

图式 7 三氟甲磺酸酐作用下的贝克曼重排

Scheme $7 \quad \mathrm{Tf}_{2} \mathrm{O}$ promoted Beckmann rearrangement

首先得到氯代亚硫酸酯中间体，该中间体质子化后，处 于反式的芳基迁移得到腈鎓离子中间体，最后水进攻该 中间体，异构化后得到产物酰胺. 该类型酰胺经水解可 以高收率地得到芳胺和羧酸衍生物, 发生了形式上的碳 碳键断裂，拓展了木质素降解的应用.

最近, Miki 课题组 ${ }^{[40]}$ 建立了一种高价碘试剂促进的 


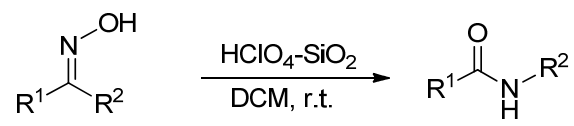<smiles></smiles>

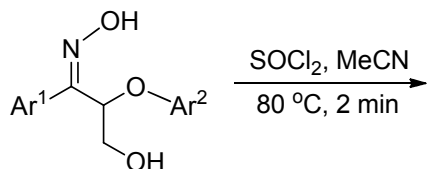<smiles>O=C(N[Al])C(CO)O[Tl]</smiles><smiles>O=S(Cl)[GeH2]O</smiles>

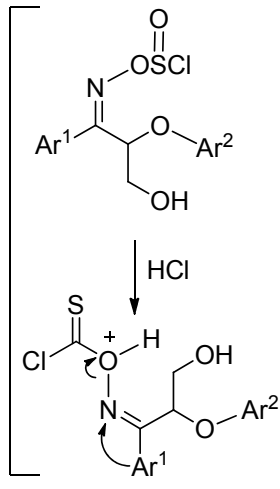<smiles>OCC(O[Al])C(O)N[Al]</smiles>

图式 8 二氯亚砜作用下的贝克曼重排

Scheme $8 \quad \mathrm{SOCl}_{2}$-mediated Beckmann rearrangement

贝克曼重排新方法(Scheme 9). 二乙酰氧基碘苯首先用 三氟化嗍乙醚进行预活化, 继而经重排得到酰胺. 该方 法底物范围较广, 反应条件温和, 无需使用昂贵的过渡 金属催化剂, 芳香和脂肪酮肟均可发生反应, 以较高的 收率得到产物酰胺.

2018 年, Hall 课题组 ${ }^{[41]}$ 报道了以硼酸和全氟频哪醇 催化的贝克曼重排, 只需 $5 \mathrm{~mol} \%$ 的硼酸在极性溶剂中 即可将酮肟转化为酰胺，该方法操作简单，条件温和， 官能团耐受性较好，二芳基、芳基烷基、二烷基肜都可 以较高的收率得到重排产物，且带有羟基、酰胺、酯基 等敏感官能团的底物也能顺利进行反应(Scheme 10). 机理验证实验表明, 反应经历了嗍酯活化、重排、肜酯 交换的串联过程.

Shia 课题组 ${ }^{[42]}$ 发展了二氯磷酸苯酯介导下酮肜的 贝克曼重排, 反应用乙腈作溶剂室温下进行, 各种不同 结构的酮肜均可以重排得到酰胺(Scheme 11). 该方法条 件温和，无需使用金属和强酸，且可以放大. 作者发现 该反应具有明显的电子效应, 苯环对位带有供电子基团

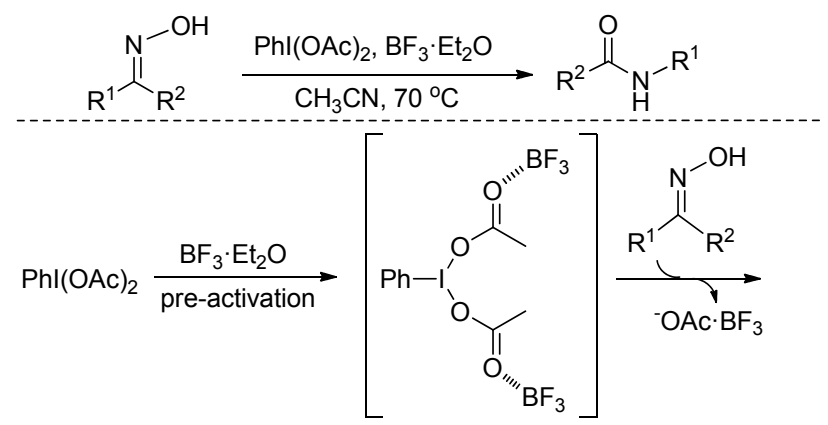

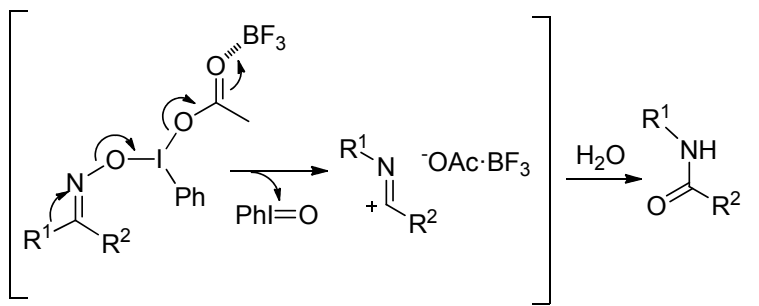

图式 9 高价碘试剂促进的贝克曼重排

Scheme 9 Hypervalent iodine reagents induced Beckmann rearrangement
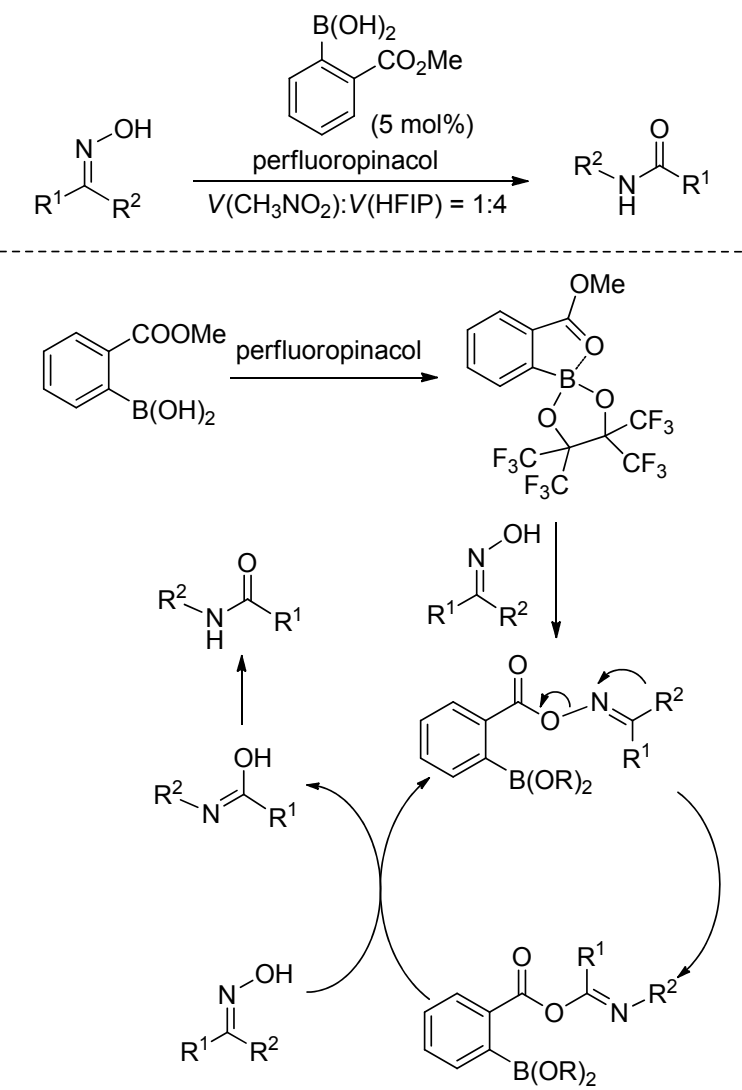

图式 10 嗍酸催化的贝克曼重排

Scheme 10 Boric acid catalyzed Beckmann rearrangement 时反应速度快且收率较高.

2018 年, Mesmaeker 课题组 ${ }^{[43]}$ 用 2,4,6-三甲基苯磺 酰羟胺作催化剂, 发展了从四元环酮出发合成 $\gamma$-内酰胺 的新方法，该方法底物范围广，单环、螺环和三环 $\gamma$-内 


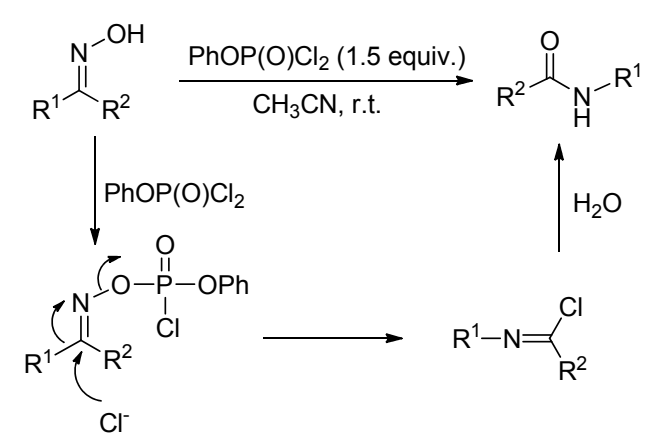

图式 11 二氯磷酸苯酯诱导的贝克曼重排

Scheme 11 Phenyl dichlorophosphate catalyzed rearrangement 酰胺均可以方便合成, 成功避免了贝克曼碎裂化. 该重 排反应的机理可能涉及四面体中间体, 而不是经典的贝 克曼重排. 原因是增加水的用量提高了反应的收率, 且 DFT 计算表明, 水可以稳定过渡态中间体 $\mathbf{A}$, 降低得到 产物酰胺的活化能(Eq. 11).<smiles>[R]C1C(=O)C([R])C1[R]</smiles><smiles>Cc1cc(C)c(S(=O)(=O)ON)c(C(C)C)c1</smiles><smiles>[R]C1NC(=O)C([R])C1[R]</smiles>

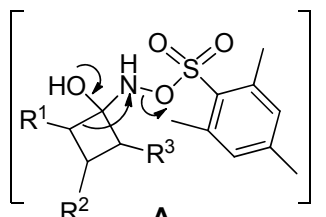

26 monocyclic, spirocyclic tricyclic examples<smiles>O=C1CC2Cc3ccccc3C2N1</smiles>

$64 \%$<smiles>O=C1CC2Cc3sccc3C2N1</smiles><smiles>CC1(C)CCCC2=C1C1NC(=O)CC1C2</smiles>

$68 \%$<smiles>O=C1CCC(c2ccc(F)cc2)N1</smiles><smiles>O=C1CC(c2ccccc2)C2(CCC2)N1</smiles>

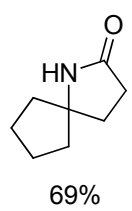

\section{2 中性条件下的贝克曼重排}

相比酸催化的重排反应, 中性条件下的贝克曼重排 条件更加温和, 底物范围更广. 2014 年, 田伟生课题 组 ${ }^{[44]}$ 用氟烷基磺酰氟作活化试剂, DBU 作碱, 实现了 $\alpha, \beta$-不饱和酮肟的贝克曼重排反应, 以较好的收率得到 烯酰胺(Eq. 12). 该反应条件温和, 操作简单, 为酸敏感 烯酰胺的制备提供了一种新的方法.

最近, Gao 课题组 ${ }^{[45]}$ 发展了以四溴化碳/三苯基膦催 化酮肜贝克曼重排的新方法, 该方法无需添加酸或金<smiles>[R]C=CC([R])=NO</smiles><smiles>O=C(/C=C/c1cccs1)N/C=C/c1cccs1</smiles><smiles>C=C(C)C1CC=C(C)NC(=O)C1</smiles>

属, 官能团耐受性好, 产率较高(Scheme 12).

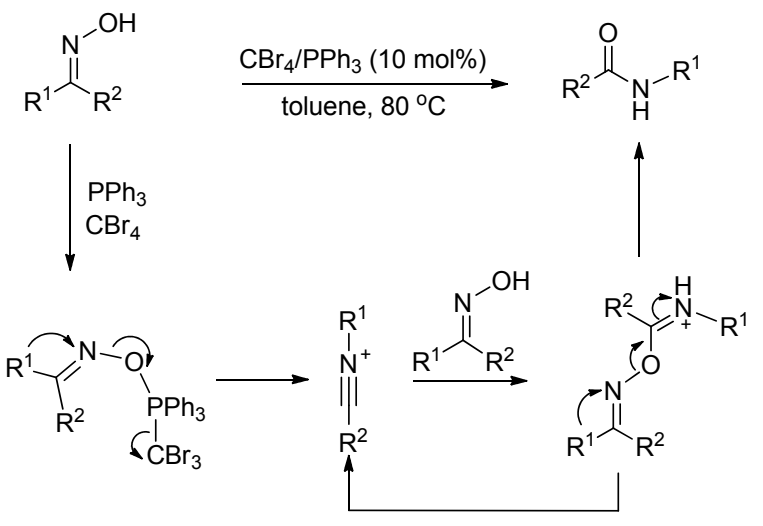

图式 12 三苯基磷催化的贝克曼重排

Scheme $12 \mathrm{PPh}_{3}$ catalyzed Beckmann rearrangement

2018 年, 郭凯课题组 ${ }^{[46]}$ 用易得的二氯咪唑啉二酮 作催化剂, 乙腈作溶剂, 建立了一种小分子催化贝克曼 重排的新方法(Scheme 13). 该方法反应时间短、底物范 围广、收率较高, 且容易放大. 作者提出并证实反应经 历了 “自我活化” 的历程 ${ }^{[47]}$. 一分子肟生成的腈鎓离子 $\mathbf{A}$ 或氯代亚胺 $\mathbf{B}$ 与另一分子肟进行反应, 得到二聚中间 体 $\mathbf{C}$, 重排后得到产物酰胺, 并重新释放出中间体 $\mathbf{A} / \mathbf{B}$, 继续进行循环.

2016 年, Mhaske 课题组 ${ }^{[48]}$ 报道了用过硫酸铵和二 甲基亚砜介导的中性条件下的贝克曼重排(Scheme 14). 该方法所用试剂便宜易得，无需使用强酸和过渡金属， 对环境友好, 底物范围广. 通过控制实验和 ${ }^{18} \mathrm{O}$ 标记实 验，验证了该反应属于自由基机理，过硫酸铵和二甲基 亚砜首先产生甲硫基自由基，接着进攻亚胺，氢转移后 分子内环化得到三元环中间体, 最后开环后得到产物.

\section{3 其他类型的贝克曼重排}

固体酸催化剂具有便宜、可回收利用、对环境友好 等优点 ${ }^{[49]}$. 2018 年, Samant 课题组 ${ }^{[50]}$ 利用固体酸 

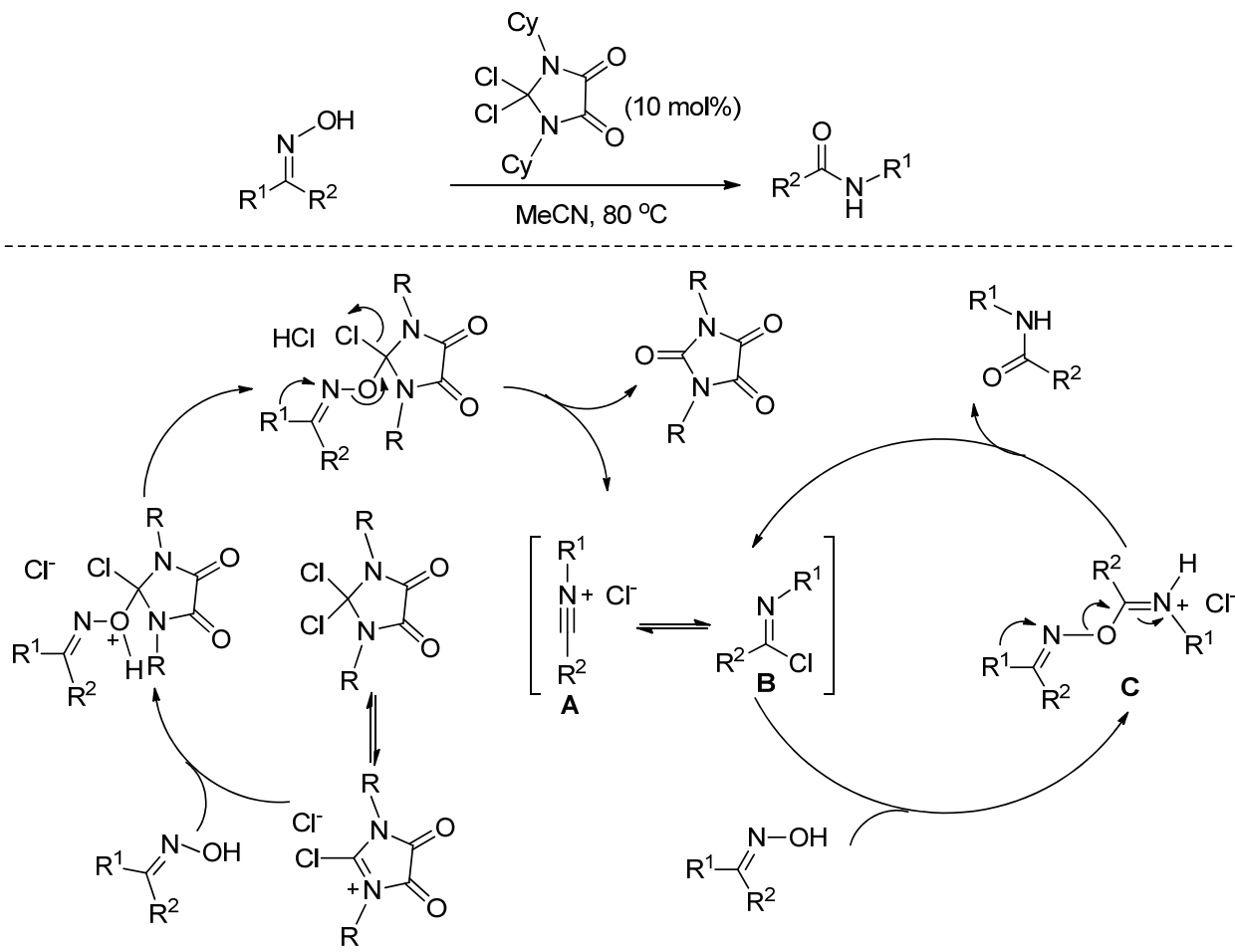

图式 13 DCIDs 催化的贝克曼重排

Scheme 13 DCIDs catalyzed Beckmann rearrangement

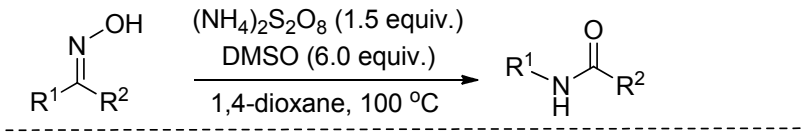

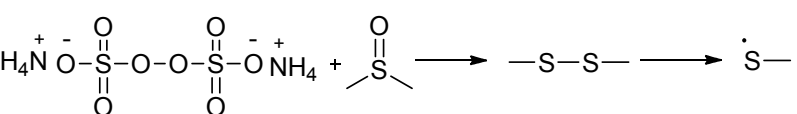

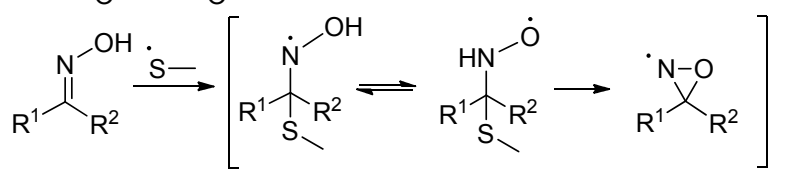
$\longrightarrow \mathrm{R}_{-\mathrm{H}}^{1} \stackrel{\mathrm{N}}{\mathrm{N}}_{\mathrm{R}^{2}}$

图式 14 过硫酸铵介导的贝克曼重排

Scheme 14 Ammonium persulfate mediated Beckmann rearrangement

$\mathrm{Fe}_{2} \mathrm{O}_{3} @ \mathrm{SiO}_{2}$ 核壳结构催化剂发展了将醛或酮一锅直接 转化为酰胺的新方法(Eq. 13). 该反应操作简单、收率 高、官能团耐受性广. 催化剂回收并重复使用五次没有 明显的活性损失. Nageswar 课题组 ${ }^{[51]}$ 使用生物甘油基碳 作为可回收固体酸催化剂, 温和条件下将各种醛直接转 化为伯酰胺.

杂多酸是通过氧原子配位桥联的含氧多酸, 是一种 酸碱性和氧化还原性兼具的双功能绿色催化剂 ${ }^{[52]}$. 2014 年, Devi 课题组 ${ }^{[53]}$ 使用磷钨酸 $\mathrm{H}_{3} \mathrm{PW}_{12} \mathrm{O}_{40}$ 作催化剂, 成
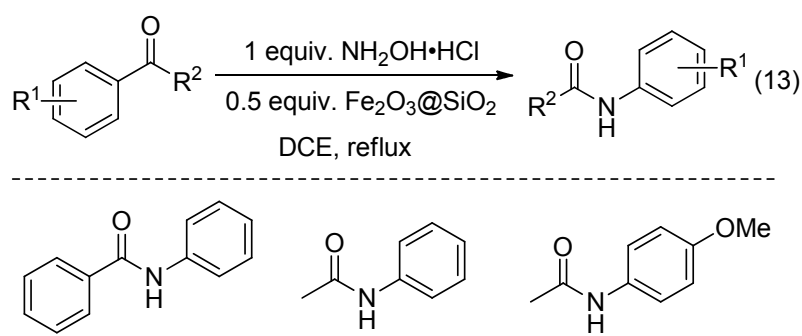<smiles>CC(=O)Nc1ccccc1</smiles><smiles>COc1ccc(NC(C)=O)cc1</smiles><smiles>COc1cc(C(N)=O)cc(OC)c1OC</smiles><smiles>NC(=O)c1ccc(Cl)cc1</smiles><smiles>NC(=O)c1ccc([N+](=O)[O-])cc1</smiles>

功催化了酮肜转化为酰胺的贝克曼重排. 该方法简单高 效且对环境友好, 催化剂便宜易得, 对水不敏感, 可回 收利用(Eq. 14).

2015 年, King 课题组 ${ }^{[54]}$ 在四乙酸铅的作用下将环 戊酮和环丁酮转化为酰氧基亚硝基中间体，之后在碱性 条件下水解生成扩环产物环羟肜酸, 一 $\mathrm{NOH}$ 基团区域 选择性地插入到取代基多的位置(Scheme 15). 酰氧基 亚硝基中间体用三苯基膦处理也可以发生贝克曼型重 排得到产物酰胺.

Singh 课题组 ${ }^{[55]}$ 发展了微波条件下三氟甲磺酸钪催 化的一锅法由醛直接合成伯酰胺的新方法, 该方法反应 时间短, 收率高. Pasha 课题组 ${ }^{[56]}$ 同样在微波辅助下实现 了 $\mathrm{SiO}_{2} \mathrm{Cl}$ 催化下醛直接合成伯酰胺的贝克曼重排，该 非均相催化剂可以回收利用. 


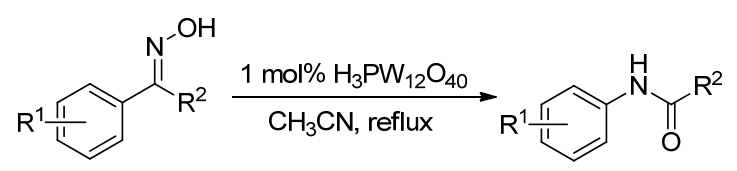<smiles>O=C1CCCCCN1</smiles>

$89 \%$<smiles>O=C(Nc1ccccc1)c1ccc(Cl)cc1</smiles>

$88 \%$<smiles>O=C1CCCCN1</smiles>

$84 \%$<smiles>CC(=O)Nc1ccc(C)cc1</smiles><smiles>CC(=O)Nc1cccs1</smiles>

$82 \%$<smiles>CC(=O)OC1(N=O)CCC1(C(=O)O[Na])C(=O)ON1C(=O)CCCC1C</smiles>

图式 15 碱性条件贝克曼重排

Scheme 15 Beckmann rearrangement under basic conditions

2015 年, Bhalla 课题组 ${ }^{[57]}$ 使用荘酰亚胺衍生物(PBI) 作为反应物和稳定剂制备得到录纳米颗粒. 该类化合物 对醛肜转化为酰胺的贝克曼重排表现出优异的催化效 率(Eq. 15).

\section{3 贝克曼重排的应用}

\section{1 贝克曼重排生成己内酰胺}

$\varepsilon$-己内酰胺作为合成尼龙-6 的起始原料, 2014 年全 球用量高达五百万吨. 传统的将环已酮肜转化为己内酰 胺的方法, 需要用到浓硫酸且产生副产物硫酸铵. 2014 年, Luo 课题组 ${ }^{[58]}$ 发展了三氟乙酸作用下环己酮肜转化 $\varepsilon$-己内酰胺的新方法, 该方法的转化率达 $100 \%$, 产率大 于 $99 \%$, 且避免了中和剂的使用及副产物硫酸铵的生 成. 最近, Tojo 课题组 ${ }^{[59]}$ 报道了一种新型的氰基胍对甲 苯磺酸盐催化剂, 可以将环己酮肜转化为己内酰胺. 该 催化剂容易制备且没有腐蚀性, 该反应不需要添加其他 促进剂，同时避免了有毒溶剂的使用(Eq. 16).

\section{1 .1 沸石(分子笁)催化}

近年来固体酸, 特别是沸石显示出对贝克曼重排优 异的催化性能, 然而传统的沸石活化位点在微孔内严重

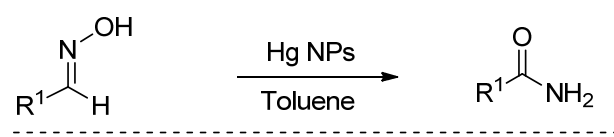<smiles>NC(=O)c1ccccc1</smiles><smiles>COc1ccc(C(N)=O)cc1</smiles><smiles>Cc1ccc(C(N)=O)cc1</smiles><smiles>NC(=O)c1ccc([N+](=O)[O-])cc1</smiles><smiles>NC(=O)c1ccc(Cl)cc1</smiles>

$76 \%$<smiles>NC(=O)c1ccccc1O</smiles><smiles>ON=C1CCCCC1</smiles><smiles>N#CN([OH+])C(N)=[NH2+]</smiles><smiles>O=C1CCCCCN1</smiles>

抑制了传质效率. 2017 年, Khayyat 课题组 ${ }^{[60]}$ 合成了一种 新型的分级硅钛沸石，该类结构包含了微孔、细孔及大 孔，具有很高的催化活性，且克服了传质效率低的缺点， 在温和条件下环已酮肜的转化率高达 $99 \%$.

使用传统的分子篎催化贝克曼重排一般需要很高 的反应温度 $\left(>350{ }^{\circ} \mathrm{C}\right)$, 最近, Raja 课题组 ${ }^{[61]}$ 设计的新 型磷酸铝 FAU 型沸石分子篮显示出优异的催化效能, 实现了在较低温度下 $\left(130{ }^{\circ} \mathrm{C}\right.$ 的贝克曼重排反应，几乎 以定量的收率得到 $\varepsilon$-己内酰胺.

沸石催化贝克曼重排的机理是内表面还是外部一 直存在争论. 最近, Deng 课题组 ${ }^{[62]}$ 通过理论计算发现, 在 $298 \mathrm{~K}$ 时, 对于环已酮弜和丙酮肜, ZSM-5 型沸石的 内表面具有较大的有效速率常数和较高的反应活性. 然 而, 在 $598 \mathrm{~K}$ 时, 对于位阻较大的环己酮肜, 沸石的孔 隙口显示出较高的反应活性.

\subsection{2 利用微反应器重排}

使用微反应器可以节能、提高反应速率，且使反应 更安全 ${ }^{[63]}$. Arai 课题组 ${ }^{[64]}$ 在无催化剂的条件下, 微反应 器中将环已酮肜经贝克曼重排转化为 $\varepsilon$-己内酰胺, 转化 率接近 $100 \%$. 2018 年, 骆广生课题组 ${ }^{[65]}$ 以三氟乙酸作 催化剂, 利用微型反应器将环已酮肜转化生成己内酰胺 (Scheme 16). 反应在 $380 \mathrm{~K}$ 的温度下 $20 \mathrm{~s}$ 即可完成, 动 力学研究表明, 环己酮肜的酯化反应和重排过程都是反 应速率的决定步骤.

\subsection{3 离子液体催化}

离子液体是一类环境友好的催化剂. 2013 年, Lee 课 题组 ${ }^{[66]}$ 报道了 $\mathrm{Pd} / \mathrm{Sc}(\mathrm{OTf})_{3} /$ 离子液体催化剂一锅法将环 己酮肟转化为 $\varepsilon$-己内酰胺的方法. 最近, Wang 课题组 ${ }^{[67]}$ 发展了一种使用铇酸钠酸性离子液体催化环己醇一锅 法转化为已内酰胺的新方法(Scheme 17). 该方法操作 


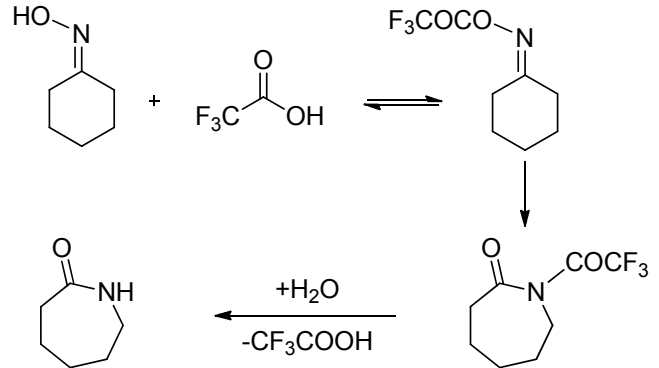

图式 16 微反应器中环己酮肜重排生成己内酰胺

Scheme 16 Rearrangement of cyclohexanone oxime to caprolactam in microreactor

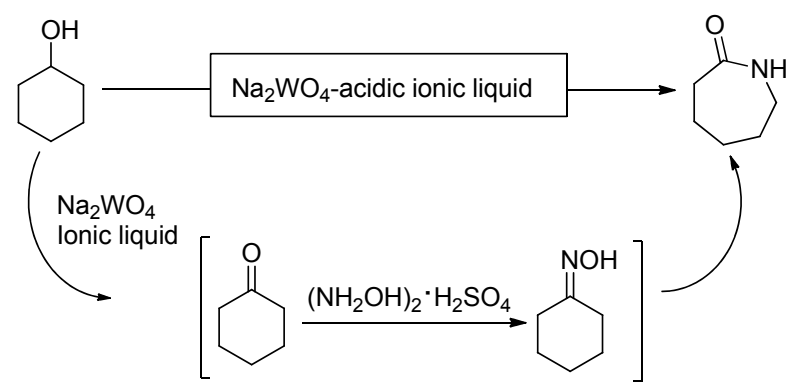

图式 17 离子液体催化的贝克曼重排

Scheme 17 Ionic liquids catalyzed Beckmann rearrangement

简便，反应条件温和，所使用的催化剂简单高效、对环 境友好、可回收利用. 作者研究发现硫酸根作为阴离子 反应效果最好, 环已醇的转化率可以达到 $97.3 \%$, 产物 己内酰胺的收率为 $76 \%$. 该方法最大的优势是将醇的氧 化、肟的制备和贝克曼重排在一锅里面完成.

\section{2 串联反应合成氮杂环化合物}

\section{2 .1 合成噁唑}

2015 年, 李兴奇课题组 ${ }^{[68]}$ 报道了用二乙胺基三氟 化硫(DAST)促进的贝克曼重排/分子内环化反应. 建立 了一种简便有效一锅法合成 5-亚氨基噁唑啉的新方法 (Eq. 17).
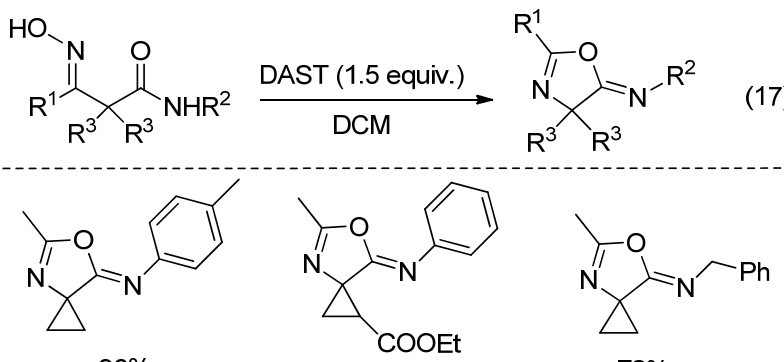

$72 \%$<smiles>CC1=NC(C)(C)C(=Nc2ccccc2)O1</smiles>

$84 \%$

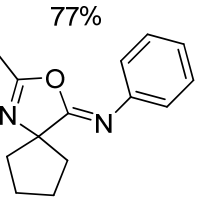

$88 \%$

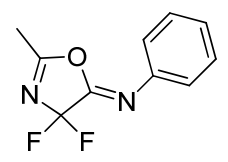

$98 \%$
2016 年, 问鹏飞课题组 ${ }^{[69]}$ 用三氟甲磺酸酐作活化
剂, DBU 作碱合成了类似的 5-亚氨基噁唑啉衍生物 (Scheme 18). 反应经历了串联的贝克曼重排/分子内环 化的过程，在室温条件下即可完成. 反应可能的途径是 酸酐首先活化肜的着基，接着贝克曼重排生成酰胺，最 后分子内的环化得到目标产物.

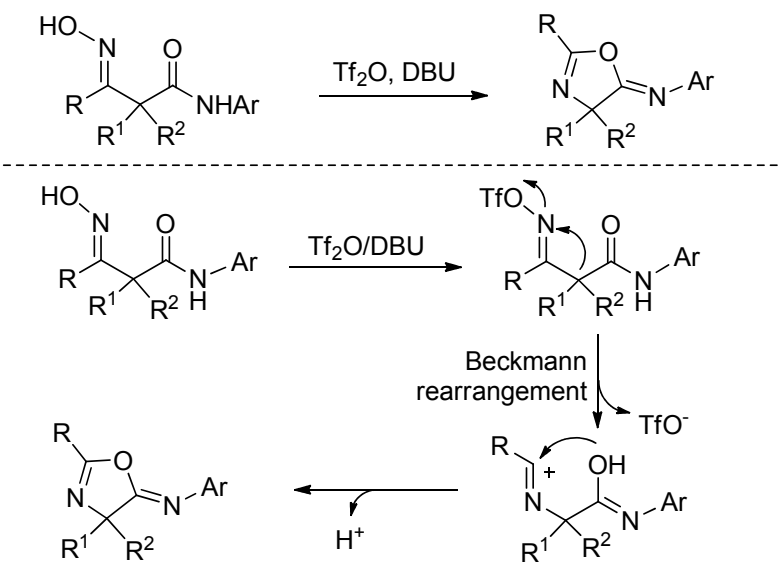

图式 18 一锅法合成噁唑啉

Scheme 18 One pot synthesis of oxazoline

2015 年, Xiong 课题组 ${ }^{[70]}$ 发展了一种用邻羟基或邻 氨基酮亚胺制备苯并噁唑和苯并咪唑衍生物的新方法 (Scheme 19). 该方法使用二乙酰氧基碘苯作为氧化剂, 引发贝克曼型 $[1,2]$ 芳基迁移，继而发生分子内环化得到 目标产物。该重排策略被成功应用于合成生物活性分子 chlormidazole 和 clemizole.

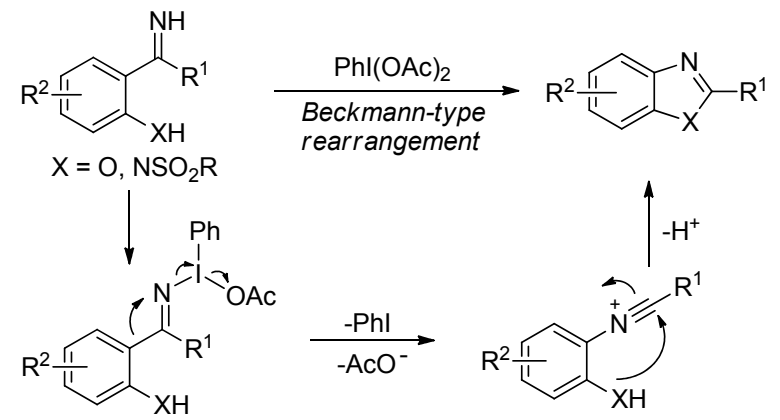

图式 19 高价碘试剂引发的贝克曼重排

Scheme 19 Beckmann rearrangement initiated by hypervalent iodine reagents

\section{2 .2 合成愳二唑}

2015 年, Tron 课题组 ${ }^{[71]}$ 以氯代肜、羟胺和异腈为原 料首先合成氨基二肜，然后将该中间体在偶氮二甲酸二 乙酯(DEAD)和三苯基膦(TPP)的作用下经串联光延反 应/贝克曼重排得到 1,2,4-恶二唑骨架. 中间体氨基二肜 的立体构型通过 X 单晶衍射确定(Scheme 20).

\section{3 天然产物及生物活性分子的合成}

2016 年, Mhaske 课题组 ${ }^{[48]}$ 用过硫酸铵和二甲基亚 砜介导的中性条件下的贝克曼重排作为关键反应，经 


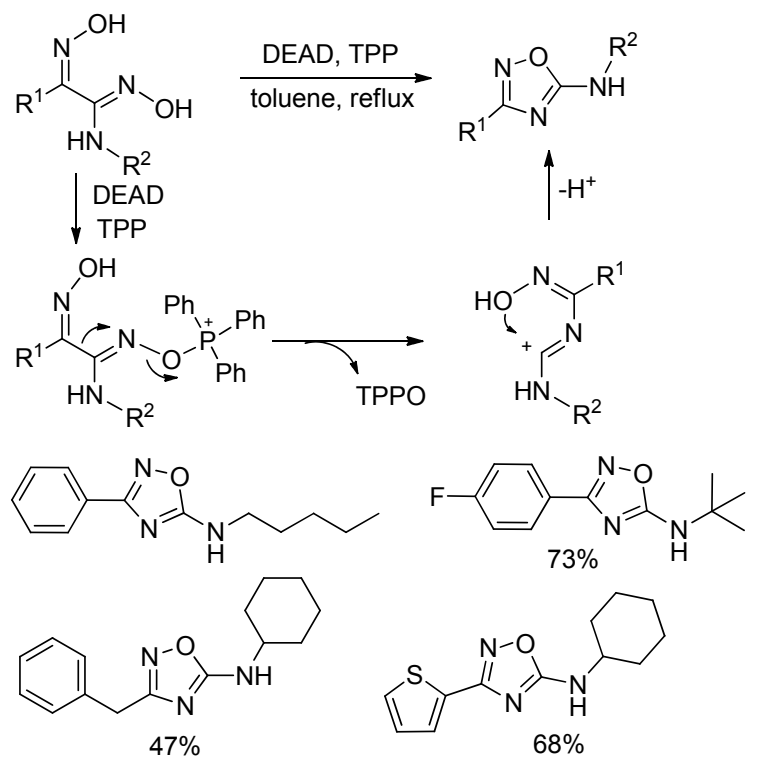

图式 20 氨基二肟合成噁二唑

Scheme 20 Synthesis of oxadiazoles by aminodioximes Pd 催化的分子内偶联反应, 成功完成了抗㾏疾天然产 物异槲皮素全合成(Scheme 21).

最近, Sperry 课题组 ${ }^{[72]}$ 通过串联的贝克曼重排/曼尼 希环化反应尝试合成氮杂草双吲哚生物碱 iheyamine A. 中间体肟经贝克曼重排得到双吲哚酰胺, 然后经分子内 Mannich 环化得到五环骨架结构, 作者尝试将其转化为 iheyamine A, 但没有成功(Scheme 22).

2017 年, 徐晖课题组 ${ }^{[73]}$ 利用二氯亚砜促进的贝克 曼重排合成了带有 7 元环内酰胺结构的鬼臼毒素类似 物, 产物结构经单晶确定(Scheme 23). 卤原子的引入对 活性影响很大, C-2'带有氯或溴原子取代的产物, 显示<smiles></smiles>

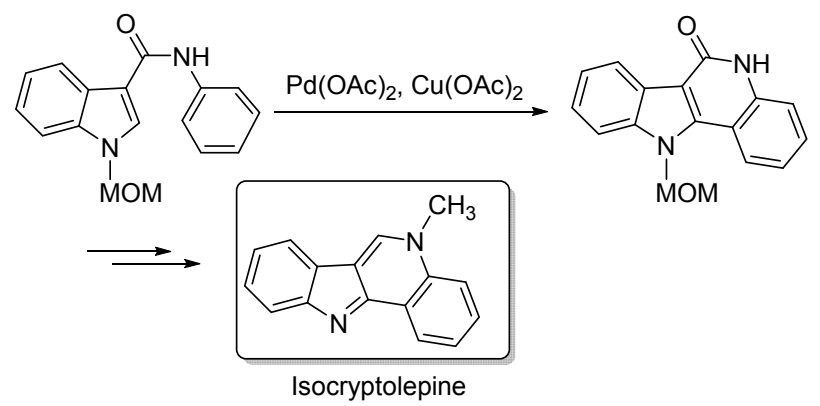

图式 21 贝克曼重排合成天然产物

Scheme 21 Synthesis of isocryptolepine through Beckmann rearrangement

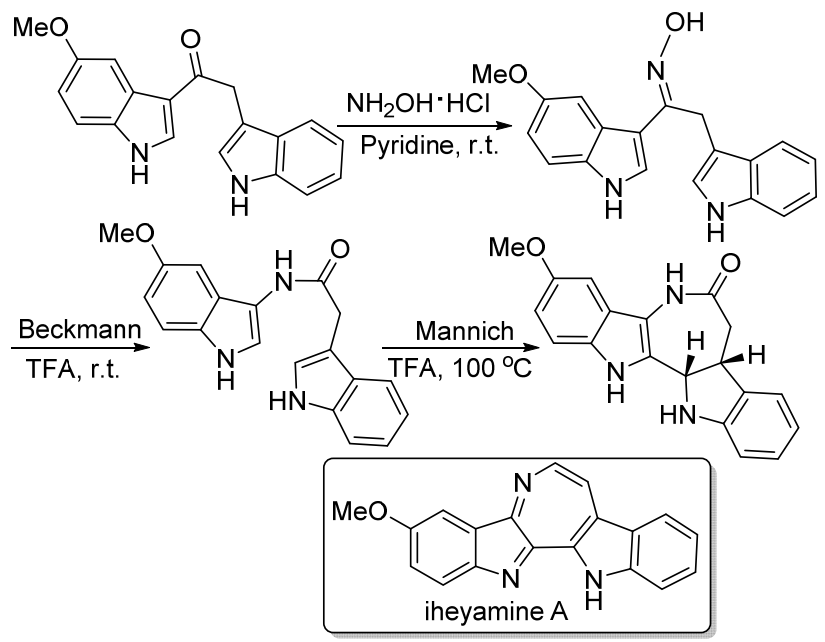

图式 22 串联的贝克曼重排/曼尼希环化反应

Scheme 22 Tandem Beckmann rearrangement/Mannich cyclization reaction<smiles>COc1cc([C@H]2c3cc4c(cc3[C@@H](O)[C@@H]3COC(=O)[C@H]23)OCO4)cc(OC)c1OC</smiles>

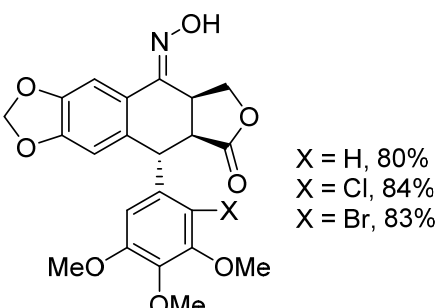

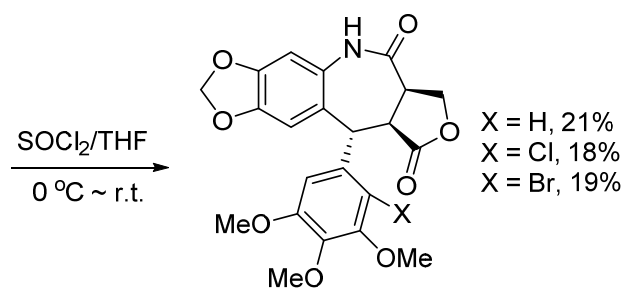<smiles>[X]c1c(C2c3cc4c(cc3/C(=N/O)C3COC(=O)C32)OCO4)cc(OC)c(OC)c1OC</smiles>

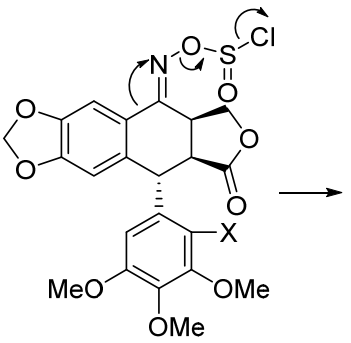<smiles>[X]c1cc([C@H]2c3cc4c(cc3N=[C+][C@@H]3COC(C)(C)C23)OCO4)cc(OC)c1OC</smiles><smiles>[X]c1cc([C@H]2c3cc4c(cc3N=C(O)C3COC(=O)[C@@H]32)OCO4)cc(OC)c1OC</smiles><smiles>[X]c1cc([C@H]2c3cc4c(OCOC)cc3NC(=O)C2COC4=O)cc(OC)c1OC</smiles>

图式 23 贝克曼重排合成杀虫剂

Scheme 23 Synthesis of insecticides by Beckmann rearrangement 
出比川楝素更好的杀黏虫活性. 同年, Mphahlele 课题 组 ${ }^{[74]}$ 利用三氟乙酸促进的贝克曼重排/三氟乙酰化反应, 合成了新型的吲哚乙酰胺衍生物. 该类结构显示出很好 的杀症原虫活性(Scheme 24).
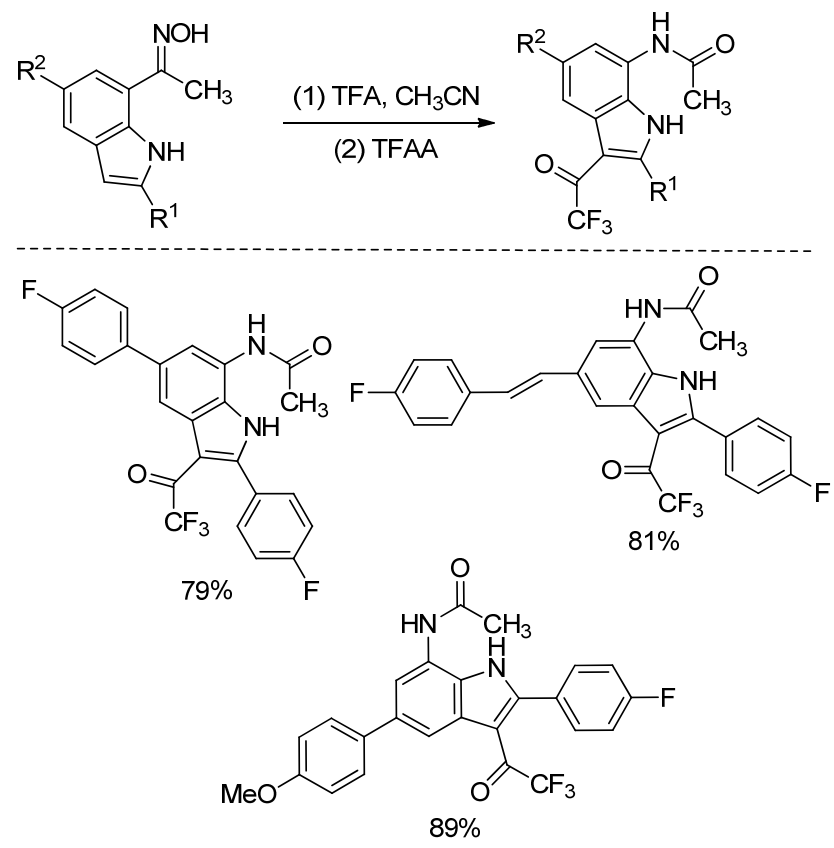

图式 24 贝克曼重排合成杀虫剂

Scheme 24 Synthesis of insecticides by Beckmann rearrangement

\section{4 总结与展望}

酰胺作为一种重要的结构单元, 在制药、材料等领 域都具有广泛的应用. 传统制备酰胺的许多策略伴随有 大量副产物或废料产生, 不符合原子经济性合成的要 求. 酮肜的贝克曼重排反应可以高效地制备 $N$-取代酰 胺, 而醛肟的重排反应是具有挑战性的, 通常得到的产 物是腈而不是酰胺. 最近几年, 金属催化的体系很好地 解决了选择性的问题, 可以高效地将醛肟转化为伯酰 胺.

综上所述, 总结了近五年来贝克曼重排的新进展, 从金属催化、无金属催化、重排的应用三个部分进行了 总结, 包括从醛、酮甚至是醇出发的一锅法串联反应. 这些新方法的发现, 丰富了贝克曼重排反应体系, 对于 进一步拓展其在医药、材料等方面的应用有着重要意义. 相信将会有更多高效、原子经济性的贝克曼重排新体系 被建立.

\section{References}

[1] (a) Greenberg, A.; Breneman, C. M.; Liebman, J. F. The Amide Linkage: Structural Significance in Chemistry, Biochemistry and Materials Science, 1st ed., Wiley, New York, 2000.

(b) Huang, P.-Q. Acta Chim. Sinica 2018, 76, 357 (in Chinese).

(黄培强, 化学学报, 2018, 76, 357.)
[2] (a) Valeur, E.; Bradley, M. Chem. Soc. Rev. 2009, 38, 606. (b) Li, Z.; Gao, B.; Huang, H. Chin. J. Org. Chem. 2018, 38, 1431 (in Chinese). (李哲健，高宝，黄汉民，有机化学，2018，38, 1431.)

[3] (a) Beckmann, E. Ber. Dtsch. Chem. Ges. 1886, 19, 988 (b) Gawley, R. E. Org. React. 1988, 35, 1.

[4] Li, Q.; Yan, L. Y.; Xia, D.; Shen, Y. C. Chin. J. Org. Chem. 2011 31, 2034 (in Chinese). (李倩, 严罗一，夏定，申永存，有机化学, 2011, 31, 2034.)

[5] Chandrasekhar, S. Comprehensive Organic Synthesis II, 2nd ed., Elsevier, Amsterdam, Vol. 7, 2014, pp. 770 800 .

[6] Crochet, P.; Cadierno, V. Chem. Commun. 2015, 51, 2495.

[7] Li, F.; Qu, P.; Ma, J.; Zou, X.; Sun C. ChemCatChem 2013, 5 , 2178.

[8] Xu, L.; Li, N. N.; Peng, H.-G.; Wu, P. ChemCatChem 2013, 5, 2462.

[9] Ali, M. A.; Punniyamurthy, T. Adv. Synth. Catal. 2010, 352, 288.

[10] Sharma, S. K.; Bishopp, S. D.; Allen, C. L.; Lawrence, R.; Bamford, M. J.; Lapkin, A. A.; Plucinski, P.; Watson, R. J.; Williams, J. M. J. Tetrahedron Lett. 2011, 52, 4252.

[11] (a) Jang, W.; Kim, S. E.; Yang, C. M.; Yoon, S.; Park, M.; Lee, J.; Kim, Y.; Kim, M. Catal. Commun. 2015, 60, 120.

(b) Yao, W.; Yu, J.; Huang, X.; Zhang, B. Chem. J. Chin. Univ 2018, 39, 926 (in Chinese).

(姚武冰, 虞姜姜, 黄相韵, 张斌, 高等学校化学学报, 2018, 39, 926.)

[12] Das, R.; Chakraborty, D. Catal. Commun. 2012, 26, 48.

[13] Allen, C. L.; Lawrence, R.; Emmett, L.; Williams, J. M. J. Adv. Synth. Catal. 2011, 353, 3262.

[14] (a) Kumar, P.; Singh, A. K.; Pandey, R.; Pandey, D. S. J. Organomet. Chem. 2011, 696, 3454.

(b) Prabhu, R. N.; Ramesh, R. RSC Adv. 2012, 2, 4515.

[15] García-Álvarez, R.; Díaz-Álvarez, A. E.; Borge, J.; Crochet, P.; Cadierno, V. Organometallics 2012, 31, 6482.

[16] García-Álvarez, R.; Díaz-Álvarez, A. E.; Crochet, P.; Cadierno, V. RSC Adv. 2013, 3, 5889

[17] García-Álvarez, R.; Zablocka, M.; Crochet, P.; Duhayon, C.; Majoral, J. P.; Cadierno, V. Green Chem. 2013, 15, 2447.

[18] Francos, J.; Menendez-Rodriguez, L.; Tomas-Mendivil, E.; Crochet, P.; Cadierno, V. RSC Adv. 2016, 6, 39044.

[19] Gonzalez-Liste, P. J.; Cadierno, V.; García-Garrido, S. E. ACS Sustainable Chem. Eng. 2015, 3, 3004.

[20] Yang, F.-L.; Zhu, X.; Rao, D.-K.; Cao, X.-N.; Li, K.; Xu, Y.; Hao, X.-Q.; Song, M.-P. RSC Adv. 2016, 6, 37093.

[21] Gowda, R. R.; Chakraborty, D. Eur. J. Org. Chem. 2011, 2226.

[22] Das, R.; Chakraborty, D. Catal. Commun. 2012, 26, 48.

[23] Mahajan, S.; Sharma, B.; Kapoor, K. K. Tetrahedron Lett. 2015, 56 , 1915.

[24] Jefferies, L. R.; Weber, S. R.; Cook, S. P. Synlett 2015, 26, 331

[25] Raju, G.; Guguloth, V.; Satyanarayana, B. RSC Adv. 2016, 6, 45036.

[26] Waskow, B.; Mano, R. A.; Giacomini, R. X.; Oliveira, D. H. Schumacher, R. F.; Wilhelm, E. A.; Luchese, C.; Savegnag, L.; Jacob, R. G. Tetrahedron Lett. 2016, 57, 5575 .

[27] Anuradha, K. S.; Layek, S.; Pathak, D. D. J. Mol. Struct. 2017, $1130,368$.

[28] Saidulu, G.; Anand, N.; Rao, K. S. R.; Burri, A.; Park, S.-E.; Burri, D. R. Catal Lett. 2011, 141, 1865.

[29] Ganguly, N. C.; Roy, S.; Mondal, P. Tetrahedron Lett. 2012, 53, 1413.

[30] Kurhe, D. K., Jayaram, R. V. Catal. Commun. 2014, 57, 69.

[31] Martínez-Asencio, A.; Yus, M.; Ramón, D. J. Tetrahedron Lett. 2012, 68, 3948.

[32] Tambara, K.; Pantoş, G. D. Org. Biomol. Chem. 2013, 11, 2466.

[33] Menéndez-Rodríguez, L.; Tomás-Mendivil, E.; Francos, J.; Nájera, C.; Crochet, P.; Cadierno, V. Catal. Sci. Technol. 2015, 5, 3754.

[34] Kiely-Collins, H. J.; Sechi, I.; Brennan, P. E.; McLaughlin, M. G. Chem. Commun. 2018, 54, 654.

[35] Xie, F.; Du, C.; Pang, Y.; Lian, X.; Xue, C.; Chen, Y.; Wang, X.; 
Cheng, M.; Guo, C.; Lin, B.; Liu, Y. Tetrahedron Lett. 2015, 57, 5820 .

[36] Rancan, E.; Aricò, F.; Quartarone, G.; Ronchin, L.; Tundo, P.; Vavasori, A. Catal. Commun. 2014, 54, 11.

[37] Kalkhambkar, R. G.; Savanur, H. M. RSC Adv. 2015, 5, 60106.

[38] Umanadh, Y.; Reddy, N. S.; Mukkanti, K.; Omprakash, G. Asian J. Chem. 2015, 27, 1209.

[39] Wang, Y.; Du, Y.; He, J.; Zhang, Y. Green Chem. 2018, 20, 3318.

[40] Oishi, R.; Segi, K.; Hamamotob, H.; Nakamuraa, A.; Maegawa, T.; Miki, Y. Synlett 2018, 29, 1465.

[41] Mo, X.; Morgan, T. D. R.; Ang, H. T.; Hall, D. G. J. Am. Chem. Soc. 2018, 140, 5264.

[42] Kuo, C.-W.; Hsieh, M.-T.; Gao, S.; Shao, Y.-M.; Yao, C.-F.; Shia, K.-S. Molecules 2012, 17, 13662.

[43] Lachia, M.; Richard, F.; Bigler, R.; Kolleth-Krieger, A.; Dieckmann, M.; Lumbroso, A.; Karadeniz, U.; Catak, S.; Mesmaeker, A. D. Tetrahedron Lett. 2018, 59, 1896.

[44] Yan, Z.; Xu, Y.; Tian, W. Tetrahedron Lett. 2014, 55, 7186.

[45] Gao, P.; Bai, Z. J. Chin. J. Chem. 2017, 35, 1673.

[46] Gao, Y.; Liu, J.; Li, Z.; Guo, T.; Xu, S.; Zhu, H.; Wei, F.; Chen, S.; Gebru, H.; Guo, K. J. Org. Chem. 2018, 83, 2040.

[47] (a) An, N.; Tian, B.-X.; Pi, H.-J.; Eriksson, L. A.; Deng, W.-P. J. Org. Chem. 2013, 78, 4297.

(b) Tian, B.-X.; An, N.; Deng, W.-P.; Eriksson, L. A. J. Org. Chem. 2013, 78, 6782 .

[48] Mahajan, P. S.; Humne, V. T.; Tanpure, S. D.; Mhaske, S. B. Org. Lett. 2016, 18, 3450.

[49] Peng, H.; Wang, D. R.; Xu, L.; Wu, P. Chin. J. Cata. 2013, 34, 2057.

[50] Jain, P. U.; Samant, S. D. ChemistrySelect 2018, 3, 1967.

[51] Ramesh, K.; Murthy, S. N.; Karnakar, K.; Reddy, K. H. V.; Nageswar, Y. V. D. Vijay, M.; Devi, B. L. A. P.; Prasad, R. B. N. Tetrahedron Lett. 2012, 53. 2636.

[52] Mao, D.; Long, Z.; Zhou, Y.; Li, J.; Wang, X.; Wang, J. RSC Adv. 2014, 4, 15635.

[53] Kaur, G.; Rajput, J. K.; Arora, P.; Devi, N. Tetrahedron Lett. 2014, $55,1136$.

[54] Hadimani, M. B.; Mukherjee, R.; Banerjee, R.; Shoman, M. E.; Aly,
O. M.; King, S. B. Tetrahedron Lett. 2015, 56, 5870.

[55] Allam, B. K.; Singh, K. N. Tetrahedron Lett. 2011, 52, 5851.

[56] Datta, B.; Pasha, M. A. Bull. Korean Chem. Soc. 2012, 33, 2129.

[57] Kaur, S.; Kumar, M.; Bhalla, V. Chem. Commun. 2015, 51, 4085.

[58] Zhang, J. S.; Riaud, A.; Wang, K.; Lu, Y. C.; Luo, G. S. Catal Lett. 2014, 144, 151.

[59] Fernandez-Stefanuto, V.; Verdia, P.; Tojo, E. New J. Chem. 2017, $41,12830$.

[60] Khayyat, S. A.; Selvin, R.; Umar, A. J. Nanosci. Nanotechnol. 2017, 17, 2170.

[61] Potter, M. E.; O’Malley, A. J.; Chapman, S.; Kezina, J.; Newland, S. H.; Silverwood, I. P.; Mukhopadhyay, S.; Carravetta, M.; Mezza, T. M.; Parker, S. F.; Catlow, C. R. A.; Raja, R. ACS Catal. 2017, 7, 2926.

[62] Chu, Y.; Li, G.; Huang, L.; Yi, X.; Xia, H.; Zheng, A.; Deng, F. Catal. Sci. Technol. 2017, 7, 2512.

[63] Zhang, J. S.; Wang, K.; Zhang, C. Y.; Luo, G. S. Chem. Eng. Process. 2016, 110, 44.

[64] Ikushima, Y.; Hatakeda, K.; Sato, O.; Yokoyama, T.; Arai, M. J. Am. Chem. Soc. 2000, 122, 1908

[65] Du, C.; Zhang, J.; Luo, G. AIChE J. 2018, 64, 571.

[66] Shin, J. Y.; Jung, D. J.; Lee, S.-G. ACS Catal. 2013, 3, 525.

[67] Wang, H.; Jia, L.; Hu R.; Gao, M.; Wang, Y. Chin. J. Catal. 2017, 38,58

[68] Li, H.; Qin, J.; Yang, Z.; Guan, X.; Zhang, L.; Liao, P.; Li, X. Chem. Commun. 2015, 51, 8637.

[69] Yu, M.; Zhang, Q.; Wang, J.; Huang, P.; Yan, P.; Zhang, R.; Dong, D. J. Chem. Sci. 2016, 128, 951.

[70] Zhang, X.; Huang, R.; Marrot, J.; Coeffard, V.; Xiong, Y. Tetrahedron 2015, 71, 700 .

[71] Mercalli, V.; Massarotti, A.; Varese, M.; Giustiniano, M.; Meneghetti, F.; Novellino, E.; Tron, G. C. J. Org. Chem. 2015, 80, 9652.

[72] Lindsay, A. C.; Sperry, J. Tetrahedron 2017, 73, 4355.

[73] Zhi, X.; Zhang, Y.; Huang, J.; Xu, H. Sci. Rep. 2017, 7, 3917.

[74] Mphahlele, M. J., Mmonwa, M. M.; Choong, Y. S. Molecules 2017, $22,1099$. 